\title{
Therapeutic activity of crude ethanolic extract of Artemisia herba alba against Trypanosoma evansi in rabbits
}

\begin{abstract}
The present work was conducted to evaluate the antitrypanosomal efficacy of crude ethanolic extract (CEE) of the aerial parts of Artemisia herba alba against Trypanosoma evansi infection in an animal model. The results indicated low levels of parasitaemia in rabbits administered with crude ethanolic extract (CEE) compared to those from the negative control group. Similarly, there was also haematologically significant difference $(\mathrm{p}<0.05)$ where low mean levels of packed cell volume (PCV) was observed in Groups 1-4 respectively. In contrast, there was no statistically significant difference in almost all investigated parameters between positive control and treatment groups of animals. In conclusion, both CEE of A. herba-alba and Berenil@ showed relatively a parasitaemia and normal haematological values in infected rabbits, thereby confirming their antiparasitic properties.
\end{abstract}

Keyword: Artemisia herba alba; Parasitaemia; Rabbits; Trypanosoma evansi 